\title{
Erratum to: Large-scale mitochondrial phylogeography in the halophilic fairy shrimp Phallocryptus spinosa (Milne-Edwards, 1840) (Branchiopoda: Anostraca)
}

\author{
Valerio Ketmaier • Daniela Pirollo • \\ Elvira De Matthaeis · Ralph Tiedemann • \\ Graziella Mura
}

Published online: 19 March 2013

(C) Springer Basel 2013

\section{Erratum to: Aquat Sci (2008) 70:65-76 \\ DOI 10.1007/s00027-007-7028-7}

The three following sequences of the mitochondrial Cytochrome Oxidase subunit I (COI) gene with GenBank Accession Numbers EU236129, EU236130 and EU236131 were erroneously assigned to the fairy shrimp Phallocryptus spinosa (haplotypes $\mathrm{L}, \mathrm{M}$ and $\mathrm{N}$ respectively in the original publication).

A BLAST search revealed that they instead match representatives of the cladoceran family Daphniidae (EU236129 and EU236131) and of the copepod family Diaptomidae (EU236130). These sequences will hence be removed from the GenBank database. We hypothesize that the cause of these erroneous amplifications has to be sought in the apparently not so rare occurrence of small crustaceans within the anostracan foliaceous limbs, as posterior microscopic inspections of a variety of ethanol-preserved anostracans confirmed (M. Ventura pers. obs.). We hence recommend to accurately checking anostracans prior to DNA extraction to exclude possible sources of contamination.

The online version of the original article can be found under doi:10.1007/s00027-007-7028-7.

\footnotetext{
V. Ketmaier $(\bowtie) \cdot R$. Tiedemann

Unit of Evolutionary Biology/Systematic Zoology,

Institute of Biochemistry and Biology, University of Potsdam,

Karl-Liebknecht-Strasse 24-25, Haus 26,

D-14476 Potsdam, Germany

e-mail: ketmaier@rz.uni-potsdam.de

V. Ketmaier - D. Pirollo - E. De Matthaeis - G. Mura

Department of Biology and Biotechnology "C. Darwin",

Universita' di Roma "La Sapienza", V.le dell'Universita 32,

I-00185 Rome, Italy
}

We phylogenetically re-analyzed the data of the original publication by using alternatively the 16S rRNA gene only (all haplotypes, including L, M, and $\mathrm{N}$ ) and the COI+16S sequences combined but excluding haplotypes $\mathrm{L}, \mathrm{M}$, and $\mathrm{N}$. Both data sets were analyzed with a Bayesian approach and the GTR $+\Gamma$ model of sequence evolution. Analytical settings were as in the original publication. Figure 1 shows the resulting trees as well as the original tree published in the original publication. In the $16 \mathrm{~S}$ tree haplotypes $\mathrm{L}$ and $\mathrm{M}$ firmly cluster together and are nested within clade IV of the original publication while haplotype $\mathrm{N}$ is placed basal to the whole aforementioned group. Clade I is confirmed. The $16 \mathrm{~S}+\mathrm{COI}$ tree is the correct tree and should substitute that presented in the original publication. It is worth noting that while the deep divergence of the erroneous clades II and III is certainly an artefact, $16 \mathrm{~S}$ data still suggest a certain degree of divergence for haplotypes $\mathrm{L}, \mathrm{M}$ and $\mathrm{N}$.

The remaining results and conclusions of the original publication are correct.

Acknowledgments We are deeply grateful to Marc Ventura Oller (CEAB, Girona, Spain) for having informed us of the mistake detailed in the present erratum. 


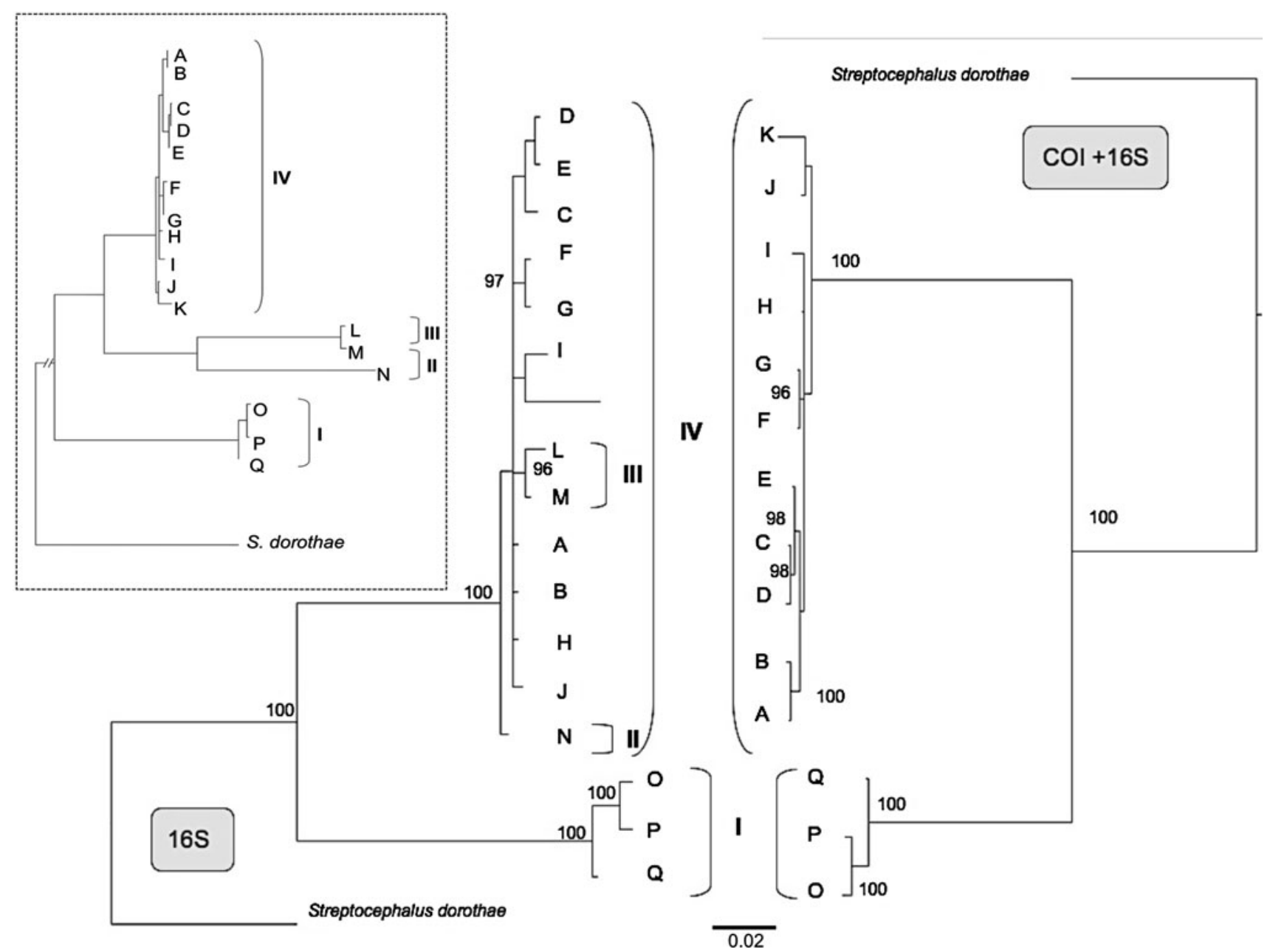

Fig. 1 Bayesian haplotype phylogram based on the GTR $+\Gamma$ model for the 16S (left including haplotypes L, M and N) and COI+16S (right haplotypes L, M and N excluded) datasets. Numbers at nodes are statistical supports for the Bayesian searches. Only nodes with a statistical support $>75 \%$ are labelled. Haplotype codes match those

in the original publication; roman numbers (I-IV) refer to the main clades identified in the original publication. The insert in the upper left corner shows a simplified version of the phylogenetic tree presented in the original paper 